\title{
Efeito do hipoclorito de cálcio na resistência de adesão do cimento obturador AH Plus à dentina
}

\author{
Effect of calcium hypochlorite on the bond strength of the \\ AH Plus sealer to dentin
}

\author{
Anelise Özkömür ${ }^{*}$ \\ Lucas Siqueira Pinheiro* \\ Júlia Eick Iglesias ${ }^{* * *}$ \\ Letícia Mestieri ${ }^{* * *}$ \\ Fabiana Soares Grecca ${ }^{* * * * *}$
}

\section{Resumo}

Objetivo: avaliar o efeito do uso de hipoclorito de cálcio $\left(\mathrm{Ca}(\mathrm{OCl})_{2}\right)$ como irrigante na resistência de adesão do cimento AH Plus (De Trey-Dentsply, Konstanz, Alemanha) à dentina pelo teste de micro push-out. Materiais e método: trinta e três dentes humanos monorradiculares foram seccionados transversalmente na junção amelocementária e divididos em três grupos: hipoclorito de sódio $(\mathrm{NaOCl}) 2,5 \%$, hipoclorito de cálcio 2,5\% e soro fisiológico. Os canais foram preparados, irrigados ao final com EDTA $17 \%$ e obturados com cones de guta percha e cimento AH Plus. Após armazenagem por 7 dias, em $100 \%$ de umidade e a $37^{\circ} \mathrm{C}$, os dentes foram seccionados transversalmente ao longo do eixo da raiz. Foram obtidas três fatias de cada dente $(n=33)$, que foram submetidas ao ensaio de push-out. O tipo de falha foi analisado por fractografia e classificado em faIha adesiva, coesiva ou mista. Os valores de resistência de união foram analisados pelo teste de Kruskal-Wallis, com nível de significância de 95\%. Resultados: o grupo $\mathrm{Ca}(\mathrm{OCl})_{2}$ 2,5\% apresentou a menor média de resistência de adesão, diferindo estatisticamente do $\mathrm{NaOCl}$ e do soro fisiológico $(p<0,05)$. Não houve diferença estatística entre os grupos irrigados com soro e $\mathrm{NaOCl}$ $(p>0,05)$. Conclusões: a falha predominante em todos os grupos foi a do tipo adesiva. $\mathrm{O} \mathrm{Ca}(\mathrm{OCl})_{2} 2,5 \%$ teve um efeito negativo sobre a força de adesão do $\mathrm{AH}$ Plus à dentina radicular quando comparado ao $\mathrm{NaOCl} 2,5 \%$.

Palavras-chave: Cimentos endodônticos. Endodontia. Hipoclorito de cálcio. Push-out. Solução irrigadora.

\section{Introdução}

A obturação do sistema de canais radiculares é uma etapa decisiva para o sucesso do tratamento endodôntico. Esse procedimento tem como objetivo promover o completo preenchimento do canal radicular, evitando penetração e fluxo de bactérias e suas toxinas para a região perirradicular ${ }^{1}$. O procedimento de obturação tem sido realizado pela utilização da guta-percha e de um cimento obturador. Como a guta-percha não possui adesão às paredes dentinárias ${ }^{2,3}$, é necessário que o cimento tenha essa importante característica. Uma alta adesão reduz a infiltração e melhora a estabilidade do material usado na obturação do canal radicular ${ }^{2}$.

$\mathrm{O}$ cimento à base de resina epóxi $\mathrm{AH}$ Plus (De Trey-Dentsply, Konstanz, Alemanha) é formado da mistura de uma pasta base (bisphenol A, óxido de ferro, sílica, óxido de zircônia e tungstato de cálcio) e uma pasta catalisadora (Dibenzil-5-oxanonane-diamina-1,9 e amina adamantada) ${ }^{4}$. Apresenta boas propriedades físicas, como estabilidade dimensional ${ }^{4,5}$, maior força de adesão à dentina quando comparado a outros cimentos endodônticos ${ }^{6}$, baixa solubilidade e desintegração ${ }^{5}$. Contudo, na presença de umidade, esse cimento não se adere eficientemente às paredes do canal ${ }^{7}$.

A solução irrigadora utilizada no preparo do canal radicular pode causar alterações nas superfícies dentárias e afetar positiva ou negativamente a adesão do material obturador ${ }^{8,9}$ e restaurador ${ }^{10}$.

\section{http://dx.doi.org/10.5335/rfo.v23i2.8306}

Cirurgiã-dentista. Mestranda em Odontologia, Faculdade de Odontologia, Universidade Luterana do Brasil, Canoas, Rio Grande do Sul, Brasil. Mestre em Odontologia, Departamento de Odontologia Conservadora, Faculdade de Odontologia, Universidade Federal do Rio Grande do Sul, Porto Alegre, Rio Grande do Sul, Brasil.

Mestre em Odontologia, Departamento de Odontologia Conservadora, Faculdade de Odontologia, Universidade Federal do Rio Grande do Sul, Porto Alegre, Rio Grande do Sul, Brasil.

Doutora em Odontologia, Departamento de Odontologia Conservadora, Faculdade de Odontologia, Universidade Federal do Rio Grande do Sul, Porto Alegre, Rio Grande do Sul, Brasil.

Doutora em Odontologia. Professora associada, Departamento de Odontologia Conservadora, Faculdade de Odontologia, Universidade Federal do Rio Grande do Sul, Porto Alegre, Rio Grande do Sul, Brasil. 
O hipoclorito de sódio $(\mathrm{NaOCl})$ tem sido utilizado como irrigante endodôntico por mais de quatro décadas devido à sua excelente ação antimicrobiana e à sua capacidade de dissolver tecido ${ }^{11}$. Porém, em altas concentrações, ele é tóxico aos tecidos periapicais ${ }^{11} \mathrm{e}$ afeta negativamente as forças de adesão de resinas compostas à dentina em restaurações na câmara pulpar após o tratamento endodôntico ${ }^{9}$. Além disso, o $\mathrm{NaOCl}$ apresenta considerável instabilidade, tornando críticos cuidados como consumo rápido e armazenamento adequado ${ }^{12}$.

Todavia, o hipoclorito de cálcio $\left[\mathrm{Ca}(\mathrm{OCl})_{2}\right]$ possui maior quantidade de cloro disponível e maior estabilidade que o $\mathrm{NaOCl}^{13}$. Historicamente, foi utilizado para a esterilização química de garrafas de leite e purificação da água ${ }^{14}$. Esse composto apresenta-se como um pó granulado branco, e a sua dissolução em água destilada pode dar origem a soluções com concentrações mais controláveis que as do $\mathrm{NaOCl}^{12}$. $\mathrm{O} \mathrm{Ca}(\mathrm{OCl})_{2}$ apresenta ainda $\mathrm{pH}$ alcalino ${ }^{12}$, capacidade de dissolução de matéria orgânica ${ }^{13}$ e atividade antimicrobiana ${ }^{15}$. O uso dessa substância tem sido considerado em endodontia ${ }^{12,13,15}$, porém seu efeito sobre a adesão dos cimentos endodônticos ainda não foi avaliado.

Dessa forma, é pertinente investigar a influência da solução de $\mathrm{Ca}(\mathrm{OCl})$, comparando-a à do $\mathrm{NaO}$ $\mathrm{Cl}$, na resistência de adesã̃o do cimento endodôntico resinoso AH Plus, por meio do teste de micro push-out. A hipótese nula é que não há diferença entre as soluções irrigadoras na resistência de adesão do cimento.

\section{Materiais e método}

O presente estudo foi aprovado pelo Comitê de Pesquisa da Faculdade de Odontologia e Ética da Universidade Federal do Rio Grande do Sul (FO-UFRGS) (CAAE 47110115.3.0000.5347).

As soluções de $\mathrm{NaOCl}$ e de $\mathrm{Ca}(\mathrm{OCl})_{2}$ foram preparadas imediatamente antes do experimento. Foram preparados $15 \mathrm{~mL}$ de $\mathrm{NaOCl}$ a partir de uma solução de $\mathrm{NaOCl}$ a $12 \%$ (Farmaquímica S.A. Produtos Químicos, Porto Alegre, Brasil), diluída em água destilada e esterilizada, de acordo com o seguinte protocolo: solução de $\mathrm{NaOCl} 2,5 \%: 3,125 \mathrm{~mL}$ de $\mathrm{NaOCl} 12 \%+11,875 \mathrm{~mL}$ de água destilada esterilizada.

Para a obtenção de $15 \mathrm{~mL}$ da solução de $\mathrm{Ca}(\mathrm{OCl})_{2}$, levando em consideração a pureza do soluto $(65 \%) \mathrm{e}$, com o objetivo de manipular uma solução de concentração precisa, a quantidade de $\mathrm{Ca}(\mathrm{OCl})_{2}$ necessária para a obtenção das soluções nas concentrações desejadas foi calculada com o auxílio de uma ferramenta desenvolvida para o preparo de soluções químicas (disponível pelo site: $<$ http://www.fop.unicamp.br/calculos/calculos/calc-porcentagem.php>), conforme segue: solução de $\mathrm{Ca}(\mathrm{OCl})_{2} 2,5 \%: 0,5769 \mathrm{~g}$ de pó de $\mathrm{Ca}(\mathrm{OCl})_{2}+15 \mathrm{~mL}$ de água destilada esterilizada.
Todas as soluções foram preparadas sob constante agitação. Após a total diluição do pó de $\mathrm{Ca}(\mathrm{OCl})_{2}$, a solução foi filtrada duas vezes para a remoção de impurezas.

Foram utilizados, neste estudo experimental in vitro, controlado, cego e aleatorizado, 33 dentes humanos monorradiculares obtidos por meio de doação após procedimento cirúrgico terapêutico indicado prévio ao estudo. Os dentes incluídos na amostra não apresentavam nenhum tratamento endodôntico prévio, sinais de reabsorção radicular ou cárie, nem canais atresiados ou curvos. Os elementos foram mantidos hidratados em água destilada em temperatura ambiente.

A coroa anatômica foi removida no nível da junção amelocementária com o auxílio de disco diamantado em baixa rotação sob constante refrigeração. As raízes foram padronizadas entre $13 \mathrm{~mm}$ e $16 \mathrm{~mm}$ de comprimento, com diâmetro apical inicial entre \#15 e \#30, e foram numeradas e divididas aleatoriamente em três grupos, onze para cada grupo, de acordo com a solução irrigadora utilizada: soro fisiológico, $\mathrm{NaOCl} 2,5 \%$ e $\mathrm{Ca}(\mathrm{OCl})_{2} 2,5 \%$.

$\mathrm{O}$ comprimento de trabalho foi definido em $1 \mathrm{~mm}$ aquém do forame apical. Primeiramente, foi feita irrigação do canal com $1 \mathrm{~mL}$ da solução teste, preparo do terço cervical (LA Axxess 20/6, SybronEndo ${ }^{\circ}$, Orange, CA, EUA) e nova irrigação com $1 \mathrm{~mL}$ da solução teste. A instrumentação foi realizada com instrumentos do tipo K-flex (Maillefer ${ }^{\circledR}$, Ballaigues, Suíça) associada à irrigação com $1 \mathrm{~mL}$ de solução teste a cada troca de instrumento. $\mathrm{O}$ instrumento \#45 foi definido como memória em todas as amostras. Um total de $10 \mathrm{~mL}$ de solução irrigadora teste foi utilizado no preparo do canal radicular. Ao final do preparo, foi feita irrigação com $1 \mathrm{~mL}$ de EDTA trissódico $17 \%$ por 3 minutos, seguido de $1 \mathrm{~mL}$ de soro fisiológico, secagem com cones de papel absorvente e seleção do cone de guta-percha.

A obturação pela técnica híbrida de Tagger foi realizada com o cimento obturador AH Plus (De Trey-Dentsply, Konstanz, Alemanha), manipulado de acordo as instruções do fabricante. Após a obturação, os dentes foram radiografados para confirmação do preenchimento do canal, selados e armazenados por 7 dias a $37^{\circ} \mathrm{C}$ em estufa com $100 \%$ de umidade relativa do ar em recipientes idênticos e codificados.

Posteriormente, o terço médio das raízes foi seccionado perpendicularmente ao seu longo eixo com o auxílio de um disco em baixa rotação (Isomet, BuehlerLtd, Lake Bluff, IL, EUA) sob refrigeração constante, obtendo-se 3 fatias, com aproximadamente $0,8 \mathrm{~mm}$ de espessura, totalizando 33 fatias para cada grupo $(n=33)$. Cada fatia foi posicionada em uma máquina de ensaios mecânicos (DL-2000, EMIC, São José dos Pinhais, Brasil). Um dispositivo cilíndrico com diâmetro de $1 \mathrm{~mm}$ foi posicionado sobre o canal obturado, na face mais apical da fatia, o qual induziu uma força no sentido ápico-cervical. 
Foi utilizada uma célula de carga de $100 \mathrm{~N}$ e velocidade de aplicação de carga de $1 \mathrm{~mm}$ por minuto. A resistência ao deslocamento do cimento para fora do canal radicular (resistência da união) foi obtida em $\mathrm{MPa}$, dividindo-se a força necessária para o deslocamento do cimento $(\mathrm{N})$ pela área adesiva $\left(\mathrm{mm}^{2}\right)$. A área adesiva foi calculada pelas fórmulas 1 e 2 :

1) $g=\left(h^{2}+(R 2-R 1)^{2}\right)^{1 / 2}(1)$

Em que:

$\mathrm{g}=$ conicidade do canal radicular

$\mathrm{h}=$ espessura da fatia

$\mathrm{R} 1$ = raio da luz radicular da face apical da raiz

$\mathrm{R} 2$ = raio da luz radicular da face cervical da raiz.

2) $A=\varpi . g .(R 1+R 2)$

Em que:

$\mathrm{A}=$ área adesiva

$\varpi=3,14$

$\mathrm{g}=$ conicidade da raiz

$\mathrm{R} 1$ = raio da luz radicular da face apical da raiz

$\mathrm{R} 2$ = raio da luz radicular da face cervical da raiz.

A espessura das fatias (h) e as medidas de $\mathrm{R} 1 \mathrm{e}$ R2 foram obtidas utilizando um paquímetro digital. Foram realizadas quatro medições do raio da luz de cada fatia, em locais diferentes, para o cálculo do raio médio dessas quatro mensurações.

Os espécimes foram observados em uma lupa estereoscópica sob magnificação de 20x (Carl Zeiss, Oberkochen, Alemanha) para determinar o padrão de fratura. Este padrão foi classificado entre coesivo (quando a fratura ocorreu apenas no cimento ou na estrutura dental), adesivo (quando a fratura ocorreu na interface estrutura dental/material obturador) ou misto (quando ocorreram os dois tipos de falhas).

Foi utilizado o teste estatístico de Kruskal-Wallis com um nível de significância de 95\%.

\section{Resultados}

Não houve diferença estatística entre os grupos soro e $\mathrm{NaOCl}$. O grupo $\mathrm{Ca}(\mathrm{OCl})_{2}$ apresentou a menor média de resistência de adesão $(\mathrm{p}<0,05)$ (Tabela 1). Em todos os grupos, a falha mais encontrada foi a adesiva (Tabela 2 ).

Tabela 1 - Média e desvio padrão do teste de push-out para os grupos (MPa)

\begin{tabular}{c|c|c}
\cline { 2 - 3 } & $\begin{array}{c}\text { Resistência média } \\
\text { (Mpa) }\end{array}$ & $\begin{array}{c}\text { Desvio } \\
\text { padrão }\end{array}$ \\
\hline $\mathrm{NaOCl} 2,5 \%+$ EDTA $17 \%$ & $3,466^{\mathrm{A}}$ & 1,999 \\
$\mathrm{Ca}(\mathrm{OCl})_{2} 2,5 \%+$ EDTA $17 \%$ & $4,226^{\mathrm{A}}$ & 2,315 \\
\hline
\end{tabular}

Nota: letras diferentes significam diferença estatística entre os grupos $(p<0,05)$.

Fonte: autores.
Tabela 2 - Porcentagem dos padrões de fratura para cada grupo

\begin{tabular}{c|c|c|c}
\cline { 2 - 4 } & Soro & $\begin{array}{c}\mathrm{NaOCl} 2,5 \% \\
+ \text { EDTA 17\% }\end{array}$ & $\begin{array}{c}\mathrm{Ca}(\mathrm{OCl})_{2} 2,5 \% \\
+ \text { EDTA } 17 \%\end{array}$ \\
\hline Adesiva & $73 \%$ & $70 \%$ & $97 \%$ \\
Coesiva & $0 \%$ & $6 \%$ & $0 \%$ \\
Mista & $27 \%$ & $24 \%$ & $3 \%$ \\
Total & $100 \%$ & $100 \%$ & $100 \%$ \\
\hline
\end{tabular}

Fonte: autores.

\section{Discussão}

A obtenção de uma obturação tridimensional, com a presença de íntimo contato entre o material obturador e a dentina, é essencial para o sucesso do tratamento endodôntico ${ }^{16}$. A utilização do cimento à base de resina epóxi $\mathrm{AH}$ Plus, juntamente com guta-percha, para a obturação tem mostrado bons resultados ao longo dos anos, tornando-o um cimento de referência para pesquisas em endodontia ${ }^{5,17}$.

Os diferentes protocolos de irrigação podem afetar a adesão de materiais obturadores à dentina $^{8,10,18}$. Além disso, a resistência de união decresce no sentido coronal para apical, devido à diminuição da densidade e do diâmetro dos túbulos dentinários, que reduz a penetração do cimento no terço apical ${ }^{19}$.

Para melhor entendimento da interação entre a dentina radicular e os materiais obturadores endodônticos, a realização de estudos avaliando a resistência de deslocamento dos materiais utilizados se torna necessária ${ }^{20,21}$. Existem diferentes métodos que podem ser usados para avaliar a adesão de um material à dentina, como teste de push-out, tenacidade de fratura e de microtração $0^{10,18,22,23}$.

$\mathrm{O}$ teste de push-out tem sido amplamente utilizado para determinar a influência das soluções irrigadoras na resistência de adesão dos cimentos endodônticos à dentina radicular, sendo um método válido para verificar como os materiais são capazes de resistir às forças de deslocamento dentro do canal radicular e classificar a qualidade dos materiais e das técnicas de obturação $0^{6,8,10,17,18,22-25}$.

Estudos anteriores utilizaram o $\mathrm{NaOCl}$ como protocolo de irrigação na resistência de push-out de cimentos endodônticos, apresentando resultados conflitantes entre eles ${ }^{10,18,22-25}$. No entanto, até o presente momento, nenhum estudo havia avaliado os efeitos da irrigação com $\mathrm{Ca}(\mathrm{OCl})_{2}$ na adesividade dos materiais obturadores às paredes dentinárias.

Collares et al. ${ }^{20}$ (2016) afirmaram que o tipo de solução irrigadora utilizado durante o tratamento endodôntico não apresenta nenhuma influência nos resultados de push-out, contrariando os estudos anteriores e os resultados encontrados no presente estudo $^{20}$.

Não há um consenso na literatura a respeito da concentração ideal de soluções de $\mathrm{NaOCl}$ a ser utilizada em endodontia ${ }^{11}$. Porém, estudos mostram que o $\mathrm{NaOCl}$ por ser um eficaz agente desproteinizante, 
causa danos aos componentes orgânicos da dentina, especialmente o colágeno, ocorrendo uma quebra da solução e liberando cloreto de sódio e oxigênio, este último sendo responsável pela inibição da polimerização interfacial de cimentos resinosos, resultando em queda dos valores de resistência de união ${ }^{26} \mathrm{e}$ diminuição da resistência mecânica da dentina ${ }^{27}$.

Ferreira et al. ${ }^{28}$ (2015) testaram a influência da utilização de $\mathrm{Ca}(\mathrm{OCl})_{2} 10$ e $15 \%$ na interface adesiva e na microinfiltração de restaurações de resina composta classe V. Quando comparado ao $\mathrm{NaOCl}$, o $\mathrm{Ca}(\mathrm{OCl})_{2}$ não apresentou diferenças quanto a desproteinização e, consequentemente, infiltração marginal das restaurações.

$\mathrm{O} \mathrm{Ca}(\mathrm{OCl})_{2}$, assim como o $\mathrm{NaOCl}$, não demonstrou ter capacidade de provocar erosão dentinária e também não consegue remover a smear layer ${ }^{29}$. Além disso, apesar de apresentar capacidade de dissolução de matéria orgânica, a velocidade de dissolução é menor do que a solução de $\mathrm{NaOCl}^{13}$, o que poderia estar relacionado a uma menor degradação das fibras colágenas, melhorando a adesividade dos cimentos.

Entretanto, no presente estudo, a utilização do $\mathrm{Ca}(\mathrm{OCl})_{2}$ como irrigante resultou em uma menor resistência de adesão do $\mathrm{AH}$ Plus à dentina, quando comparada ao $\mathrm{NaOCl}$ e ao soro, rejeitando a hipótese nula. Esse resultado pode estar associado com o precipitado deixado pela solução de $\mathrm{Ca}(\mathrm{OCl})_{2}{ }^{12-14,28}$.

Tully $^{14}$ (1914) relatou que o $\mathrm{Ca}(\mathrm{OCl})_{2}$, quando dissolvido em água, deixa um resíduo composto principalmente por hidrato de cálcio e carbonato de cálcio. Leonardo et al. ${ }^{12}$ (2016) observaram a formação de um precipitado no fundo das embalagens utilizadas para armazenar as soluções de $\mathrm{Ca}(\mathrm{OCl})_{2}$. Esse precipitado foi enviado para análise por espectroscopia por dispersão de energia (EDS), que detectou que as partículas presentes nesse precipitado eram predominantemente cálcio ${ }^{12}$. Utilizando a mesma análise, Ferreira et al. ${ }^{28}$ (2015) também observaram a presença de cálcio depositado sobre a superfície dentinária.

Nenhum estudo revelou se o precipitado encontrado em soluções de $\mathrm{Ca}(\mathrm{OCl})_{2}$ pode ser facilmente removido com soluções inertes, como água destilada ou soro fisiológico. A presença de um precipitado no interior do canal radicular, que não é removido com facilidade, poderia promover o vedamento dos túbulos dentinários, impedindo, assim, a correta penetração e a adesão do cimento à superfície dentinária. Essa hipótese poderia explicar por que o grupo irrigado com $\mathrm{Ca}(\mathrm{OCl})_{2}$ apresentou uma menor resistência de adesão em comparação aos grupos que foram irrigados com $\mathrm{NaOCl}$ e soro fisiológico.

Estudos que avaliam a resistência adesiva de um determinado material a um substrato devem realizar a análise do tipo de falha $a^{6,8,10,18,23,25}$. No presente estudo, foi utilizado o método da fractografia $^{8,18,26}$, que é geralmente determinada por análise qualitativa visual, para verificar se a fratura foi adesiva, coesiva ou mista. Sendo assim, foi observado que as amostras do $\mathrm{Ca}(\mathrm{OCl})_{2}$ apresentaram, quase que na sua totalidade, fratura adesiva, indicando nível inadequado de adesão entre o cimento e a dentina. Já nos grupos de $\mathrm{NaOCl}$ e de soro, foram constatados cerca de $30 \%$ de fraturas mistas e coesivas, o que explica os graus mais altos de adesão do $\mathrm{AH}$ Plus à dentina radicular.

\section{Conclusões}

Diante da metodologia utilizada neste estudo in vitro, observou-se que o hipoclorito de cálcio 2,5\% teve um efeito negativo sobre a força de adesão do $\mathrm{AH}$ Plus à dentina radicular quando comparado ao hipoclorito de sódio $2,5 \%$, podendo comprometer o selamento. Mais estudos devem ser realizados a fim de avaliar a influência dos sedimentos encontrados em soluções de $\mathrm{Ca}(\mathrm{OCl})_{2}$.

\section{Abstract}

Objective: to evaluate the effect of calcium hypochlorite $\left(\mathrm{Ca}(\mathrm{OCl})_{2}\right)$ as an irrigant on the bond strength of the AH Plus sealer (De Trey-Dentsply, Konstanz, Germany) to dentin, using the micro push-out test. Materials and method: thirty-three single-rooted human teeth were cross-sectioned on the cementoenamel junction and divided into three groups: $2.5 \%$ sodium hypochlorite ( $\mathrm{Na}$ $\mathrm{OCl}), 2.5 \%$ calcium hypochlorite, and saline solution. The canals were prepared, irrigated with 17\% EDTA at the end, and filled with gutta-percha cones and AH Plus sealer. After being stored for seven days at 100\% humidity and $37^{\circ} \mathrm{C}$, the teeth were cross-sectioned along the root axis. Three slices of each tooth $(n=33)$ were obtained and subjected to the push-out test. Failure mode was analyzed by fractography and classified as adhesive, cohesive, or mixed. Bond strength values were analyzed by the Kruskall-Wallis test at 95\% significance level. Results: the $2.5 \% \mathrm{Ca}(\mathrm{OCl})$, group showed the lowest bond strength mean, differing statistically from $2.5 \% \mathrm{NaOCl}$ and saline solution $(p<0.05)$. There was no statistical difference between the groups irrigated with saline solution and $\mathrm{NaOCl}(\mathrm{p}>0.05)$. Conclusion: the adhesive failure was predominant in all groups. The $2.5 \% \mathrm{Ca}(\mathrm{OCl})$, had a negative effect on the bond strength of $\mathrm{AH}$ Plus to the root dentin when compared to $2.5 \% \mathrm{NaOCl}$.

Keywords: Endodontic sealers. Endodontics. Calcium hypochlorite. Push-out. Irrigating solution.

\section{Referências}

1. von Fraunhofer JA, Fagundes DK, Mc Donald NJ, Dumsha TC. The effect of root canal preparation on microleakage within endodontically treated teeth: an in vitro study. Int Endod J 2000; 33(4):355-60.

2. Saleh IM, Ruyter IE, Haapasalo M, Orstavik D. The effects of dentine pretreatment on the adhesion of root-canal sealers. Int Endod J 2002; 35(10):859-66. 
3. Orstavik D. Materials used for root canal obturation: technical, biological and clinical testing. Endod Topics 2005; 12(1):25-38.

4. Lee KJ, Kwak SW, Ha JH, Lee WC, Kim HC. Physicochemical properties of epoxy resin-based and bioceramic-based root canal sealers. Bioinorg Chem Appl 2017; 2017:2582849.

5. Versiani MA, Carvalho-Junior JR, Padilha MIAF, Lacey S, Pascon EA, Sousa-Neto MD. A comparative study of physicochemical properties of AH Plus and Epiphany root canal sealants. Int Endod J 2006; 39(6):464-71.

6. Ersahan S, Aydin C. Dislocation resistance of iRoot SP, a calcium silicate-based sealer, from radicular dentine. J Endod 2010; 36(12):2000-2.

7. Roggendorf MJ, Ebert J, Petschelt A, Frankenberger R. Influence of moisture on the apical seal of root canal fillings with five different types of sealer. J Endod 2007; 33(1):31-3.

8. Shokouhinejad N, Sharifian MR, Jafari M, Sabeti MA. Pushout bond strength of Resilon/Epiphany self-etch and guttapercha/AH26 after different irrigation protocols. Oral Surg Oral Med Oral Pathol Oral Radiol Endod 2010; 110(5):e88-e92.

9. Neelakantan P, Varughese AA, Sharma S, Subbarao CV, Zehnder M, De-Deus G. Continuous chelation irrigation improves the adhesion of epoxy resin-based root canal sealer to root dentine. Int Endod J 2012; 45(12):1097-102.

10. Ozturk B, Özer F. Effect of $\mathrm{NaOCl}$ on Bond Strengths of Bonding Agents to Pulp Chamber Lateral Walls. J Endod 2004; 30(5):362-5.

11. Zehnder M. Root canal irrigants. J Endod 2006; 32(5):38998.

12. Leonardo NG, Carlotto IB, Luisi SB, Kopper PM, Grecca FS, Montagner F. Calcium hypochlorite solutions: evaluation of surface tension and effect of different storage conditions and time periods over $\mathrm{pH}$ and available chlorine content. J Endod 2016 ; 42(4):641-5.

13. Dutta A, Saunders WP. Comparative evaluation of calcium hypochlorite and sodium hypochlorite on soft-tissue dissolution. J Endod 2012; 38(10):1395-8.

14. Tully EJ. Study of calcium hypochlorite as a disinfectant of water. Am J Public Health (NY) 1914; 4(5):423-35.

15. De Almeida AP, Souza MA, Miyagaki DC, Dal Bello Y, Cecchin D, Farina AP. Comparative evaluation of calcium hypochlorite and sodium hypochlorite associated with passive ultrasonic irrigation on antimicrobial activity of a root canal system infected with Enterococcus faecalis: an in vitro study. J Endod 2014; 40(12):1953-7.

16. Schilder H. Filling root canals in three dimensions. J Endod 2006; 32(4):281-90.

17. Mishra P, Sharma A, Mishra S, Gupta M. Push-out bond strength of different endodontic obturation material at three different sites - in-vitro study. J Clin Exp Dent 2017; 9(6):e733-7.

18. Stelzer R, Schaller HG, Gernhardt CR. Push-out bond strength of realseal SE and AH Plus after using different irrigation solutions. J Endod 2014; 40(10):1654-7.

19. Hedge V, Arora S. Effect of advanced irrigation protocols on self-expanding Smart-Seal obturation system: a scanning electron microscopic push-out bond strength study. Contemp Clin Dent 2015; 6(1):26-30.

20. Collares FM, Portella FF, Rodrigues SB, Celeste RK, Leitune VC, Samuel SM. The influence of methodological variables on the push-out resistance to dislodgement of root filling materials: a meta-regression analysis. Int Endod J 2016; 49(9):836-49.
21. Neelakantan P, Ahmed HMA, Wong MCM, Matilinna JP, Cheung GSP. Effect of root canal irrigation protocols on the dislocation resistance of mineral trioxide aggregate-based materials: a systematic review of laboratory studies. Int Endod J 2018; 51(8):847-61.

22. Nunes VH, Silva RG, Alfredo E, Sousa-Neto MD, Silva-Souza YTC. Adhesion of Epiphany and AH Plus Sealers to human root dentin treated with different solutions. Braz Dent J 2008; 19(1):46-50.

23. Prado M, Simão RA, Gomes BPFA. Effect of different irrigation protocols on resin sealer bond strength to dentin. $\mathrm{J}$ Endod 2013; 39(5):689-92.

24. Rocha AW, De Andrade CD, Leitune VC, Collares FM, Samuel SM, Grecca FS, et al. Influence of endodontic irrigants on resin sealer bond strength to radicular dentin. Bull Tokyo Dent Coll 2012; 53(1):1-7.

25. Baldissera R, Rosa RA, Wagner MH, Kuga MC, Grecca FS, Bodanezi A, et al. Adhesion of real seal to human root dentin treated with different solutions. Braz Dent J 2012; 23(5):5216.

26. Ari H, Yasar E, Belli S. Effects of $\mathrm{NaOCl}$ on bond strengths of resin cements to root canal dentin. J Endod 2003; 29(4):24851.

27. Cecchin D, Giaretta VS, Cadorin BG, Souza MA, Vidal CMP, Farina AP. Effect of synthetic and natural-derived novel endodontic irrigant solutions on mechanical properties of human dentin. J Mater Sci: Mater Med 2017; 28(9):141.

28. Ferreira MBC, Carlini Junior B, Galafassi D, Gobbi DL. Calcium Hypochlorite as a Dentin Deproteinization Agent: Microleakage, Scanning Electron Microscopy and Elemental Analysis. Microsc Res Tech 2015; 78(8):676-81.

29. Görduysus M, Küçükkaya S, Bayramgil NP, Görduysus ÖM. Evaluation of the effects of two novel irrigants on intraradicular dentine erosion, debris and smear layer removal. Restor Dent Endod 2015; 40(3):216-22.

\section{Endereço para correspondência:}

Fabiana Soares Grecca

Faculdade de Odontologia, Universidade Federal do Rio Grande do Sul

Rua Ramiro Barcelos, 2.492, Bairro Santana

90035-00, Porto Alegre, RS, Brasil

Telefones: (51) 33085191 - (51) 33085010

E-mail: fabiana.grecca@ufrgs.br

Recebido: 25/06/18. Aceito: 31/07/18. 